\title{
Instantaneous monitoring of heart beat dynamics during anesthesia and sedation
}

\author{
Gaetano Valenza ${ }^{1,2,4}$, Oluwaseun Akeju 1,2, Kara J Pavone ${ }^{1}$, Luca Citi ${ }^{1,5}$, Katharine E Hartnack ${ }^{1}$, Aaron Sampson ${ }^{1}$, \\ Patrick L Purdon ${ }^{1,2,3}$, Emery N Brown $n^{1,2,3}$ and Riccardo Barbieri ${ }^{1,2,3^{*}}$
}

\author{
* Correspondence: \\ barbieri@neurostat.mit.edu \\ ${ }^{1}$ Department of Anesthesia, Critical \\ Care and Pain Medicine, \\ Massachusetts General Hospital, 55 \\ Fruit Street, Jackson 4, Boston, MA \\ 02114, USA \\ ${ }^{2}$ Harvard Medical School, 25 Shattuck \\ Street, Boston, MA 02115, USA \\ Full list of author information is \\ available at the end of the article
}

\begin{abstract}
Anesthesia-induced altered arousal depends on drugs having their effect in specific brain regions. These effects are also reflected in autonomic nervous system (ANS) outflow dynamics. To this extent, instantaneous monitoring of ANS outflow, based on neurophysiological and computational modeling, may provide a more accurate assessment of the action of anesthetic agents on the cardiovascular system. This will aid anesthesia care providers in maintaining homeostatic equilibrium and help to minimize drug administration while maintaining antinociceptive effects. In previous studies, we established a point process paradigm for analyzing heartbeat dynamics and have successfully applied these methods to a wide range of cardiovascular data and protocols. We recently devised a novel instantaneous nonlinear assessment of ANS outflow, also suitable and effective for real-time monitoring of the fast hemodynamic and autonomic effects during induction and emergence from anesthesia. Our goal is to demonstrate that our framework is suitable for instantaneous monitoring of the ANS response during administration of a broad range of anesthetic drugs. Specifically, we compare the hemodynamic and autonomic effects in study participants undergoing propofol (PROP) and dexmedetomidine (DMED) administration. Our methods provide an instantaneous characterization of autonomic state at different stages of sedation and anesthesia by tracking autonomic dynamics at very high time-resolution. Our results suggest that refined methods for analyzing linear and nonlinear heartbeat dynamics during administration of specific anesthetic drugs are able to overcome nonstationary limitations as well as reducing inter-subject variability, thus providing a potential real-time monitoring approach for patients receiving anesthesia.
\end{abstract}

Keywords: Autonomic nervous system; Electrocardiogram; Electroencephalogram; Heart rate variability; Respiratory sinus arrhythmia; Anesthesia; Sedation; Propofol; Dexmedetomidine; Instantaneous point process monitoring

\section{Background}

Despite recent technological advances in anesthetic delivery and monitoring systems and the growing body of information on molecular mechanisms of anesthetic actions, most anesthesia care providers monitor drug-induced altered states of arousal with basic clinical signs (e.g., heart rate, blood pressure). Due to the high prevalence of anesthesia-related morbidity, more precise monitoring tools are required. In particular, the administration of anesthetic agents can result in hypotension, hypoxia, and cardiac dysrhythmias. Post-operative recall of intraoperative events, including sleep

\section{Springer}


disturbances, dreams, nightmares, flashbacks and anxiety, as well as post-traumatic stress disorder [1], is also an important source of anesthesia-related morbidity. It is estimated that the incidence of awareness under anesthesia is experienced by 20,000 to 40,000 individuals a year in the United States [2]. On the other hand, small surgical operations, as well as most nonsurgical procedures, do not require loss of consciousness and may be performed without discomfort using reduced doses of anesthetic agents than are currently used.

It is known that anesthesia-induced altered states of arousal depend on drugs having their effect in specific brain regions. For instance, propofol (PROP) is a GABA-A agonist that primarily targets pyramidal neurons in the lower output layers of the cortex. It elicits reductions in cortical and subcortical responses to auditory and noxious stimuli. In particular, progressive failure to perceive or respond to auditory or noxious stimuli is associated with a reduction in functional connectivity between the putamen and other brain regions [3]. Varying the concentration of propofol differentially modulates brain activation [4,5], suggesting that the effects on the brain are also likely reflected in autonomic nervous system (ANS) outflow dynamics. Dexmedetomidine (DMED) is an $\alpha 2$ adrenergic agonist that binds to the receptors in the locus ceruleus with the downstream effect leading to activation of the ventrolateral preoptic nucleus (VLPO). The active VLPO facilitates GABA-A and galanin-mediated inhibition of the midbrain, hypothalamic, and pontine arousal nuclei, promoting altered arousal [6]. It is well established that changes in neurophysiological recordings such as the electroencephalogram (EEG) can provide a reliable, empirical characterization of anesthesia-induced loss of consciousness $[7,8]$. Systematic EEG changes have long been known to occur at different levels of intravenous and inhaled anesthetics, including increases in frontal EEG power [9-15], shifting of the power spectrum towards lower frequencies [16], changes in coherence [11,17], and in deep anesthesia, burst-suppression and isoelectricity [18]. These developed metrics could be used in conjunction with ANS measures for a novel multimodal assessment of ANS reactions to pain during sedation.

The ANS is known to regulate physiological homeostasis and the organism's response to environmental and psychological stressors. Innervation of the heart from the ANS sympathetic branch causes increases in heart rate and vascular constriction. Conversely, the vagus nerve, the main parasympathetic innervation of the heart, causes a decrease in heart rate. Anesthetic agents target important brain areas involved in the central autonomic network, particularly the brainstem centers processing and integrating information to generate ANS outflow to multiple organs [6]. As a consequence, virtually every drug used for sedation has an effect on the ANS, a critical factor to take into account when assessing nociceptive levels. The effect of anesthetic agent on the ANS varies due to the different neural mechanisms of anesthetic action [6]. For instance, propofol attenuates autonomic tone and baroreflex responses to hypotension resulting in a decrease in blood pressure that is largely due to vasodilatation [19-22]. On the other hand, dexmedetomidine reduces systemic sympathetic tone without altering baroreflex sensitivity [22-31].

Heart rate variability (HRV) is conventionally defined as the variation in instantaneous heart rate around its mean [32]. It is considered to be an important quantitative 
marker of cardiovascular regulation by the ANS [32]. Frequency analysis of HRV allows for the estimation of the balance between the sympathetic and the parasympathetic nervous systems. The specific type of general anesthetic agent used has been shown to have a differential effect on the change of HRV when measured after induction of general anesthesia [33]. A recent review article by Mazzeo and colleagues [34] summarizes some of the most relevant findings and limits of HRV as a diagnostic and prognostic tool in anesthesia and concludes that investigation of HRV as a method of monitoring the depth of anesthesia, assessing the response to painful stimuli, did not yield uniform results and needs more extensive investigations.' Several other important studies have considered HRV to quantify anesthesia [35-51], demonstrating the usefulness of HRV measures. In particular, time-varying identification methods have provided successful characterization of PROP and DMED effects on the ANS [19,20,24,28,44,51]. Several other studies have considered ANS measures in an attempt to monitor pain during anesthesia [52-63]. However, ANS measures have yet not been able to provide strong predictive power due to high inter-individual variability and large random variations within subjects. Due to differences in study populations and settings, and important limitations in accounting for confounders, such as assisted ventilation, compensative drug administration, or surgical stimulation, a comprehensive comparative assessment of the proposed parameters is indeed quite problematic.

The presented work is aimed at combining novel instantaneous measures of autonomic activity to provide instantaneous monitoring of the ANS response with sedation. In particular, we have devised a novel assessment of ANS outflow based on instantaneous measures of autonomic tone able to track fast hemodynamic and autonomic effects during induction and emergence from altered arousal. In previous studies, we established a paradigm for using point process methods for analyzing heartbeat dynamics $[64,65]$. We have also assessed sympathovagal indices of heart rate variability, as well as baroreflex and respiratory sinus arrhythmia [66-70], and we have successfully applied these methods to protocols including induction and emergence from anesthesia [71-75]. We have further identified specific measures of complexity and nonlinearity associated with loss of consciousness during anesthesia [75]. Most importantly, these methods can track autonomic dynamics in real time. In this study, we present exemplary analyses from a multimodal (EEG, ECG, respiration) characterization in study participants undergoing PROP and DMED administration. Furthermore, in order to validate the proposed algorithms' ability to track pharmacological interventions in the operating room (OR) or the intensive care unit (ICU), we focus on how specific features of our framework are able to characterize instantaneous signatures of the hemodynamic and autonomic effects of the anesthetics before and during loss of consciousness.

\section{Methods}

\section{Experimental protocol}

We have considered experimental datasets from healthy volunteer subjects participating in two studies approved by the Massachusetts General Hospital (MGH) Institutional Review Board.

Study 1 Propofol was intravenously infused in six healthy subjects using a computercontrolled delivery system running STANPUMP connected to a Harvard 22 syringe pump (Harvard Apparatus, Holliston, MA, USA). Five effect-site 
target levels (L1 to L5, step propofol concentration increase of $5 \mathrm{mcg} / \mathrm{ml}$ ) were each maintained for $15 \mathrm{~min}$, respectively, and then step-wise decreased by $5 \mathrm{mcg} / \mathrm{ml}$ per epoch during E6, E7, and E8 before full emergence from anesthesia. Along the experiment, subjects listened to pre-recorded auditory stimuli and were instructed to press a button to differentiate between sounds. We used the loss of button responses as a marker of loss of consciousness (LOC). EEG, capnography, pulse oximetry, ECG, and arterial BP were recorded and monitored continuously at $500 \mathrm{~Hz}$ throughout the study. Study participants were also fitted with a respiration belt and a piezo-electric pulse transducer for monitoring. Bag-mask ventilation with 30\% oxygen was administered as needed in the event of propofol-induced apnea. Because propofol is a potent peripheral vasodilator, phenylephrine was administered intravenously to maintain mean arterial BP within 20\% of the baseline when needed. This first study includes results from baseline and increasing propofol concentration levels of two male and four female subjects between 20 and 32 years old.

Study 2 Dexmedetomidine was intravenously infused in six healthy subjects using a Medfusion 3500 syringe infusion pump (Smiths Medical, Dublin, OH, USA). EEG, capnography, pulse oximetry, and ECG were recorded and monitored continuously during the study protocol. EEG was sampled at 1,000 Hz, ECG at $240 \mathrm{~Hz}$. In specific instances, respiration belts were used to record lung volume changes at $240 \mathrm{~Hz}$. The dexmedetomidine infusion started with a loading dose of $1 \mathrm{mcg} / \mathrm{kg}$ over $10 \mathrm{~min}$ followed by an intravenous infusion of dexmedetomidine to be maintained at a maximum of $0.7 \mathrm{mcg} / \mathrm{kg} / \mathrm{h}$. This second study includes results from the first $28 \mathrm{~min}$ of administration of three male and three female subjects between 21 and 29 years old.

\section{Preprocessing analysis}

$\mathrm{R}$ wave peaks are detected from the ECG signal using an automatic algorithm. The $\mathrm{R}$ wave markers are tested to eliminate artifacts and correct eventual undetected beats or beats erroneously detected by the automatic peak detection procedure. Ectopic beat classification is also carried out with our specifically tailored routines [76]. Respiration, when recorded, is low-pass filtered at $10 \mathrm{~Hz}$.

\section{Point process assessment of HRV}

The $\mathrm{R}$ wave events are a sequence of discrete occurrences in continuous time and hence, they can be analyzed meaningfully and continuously monitored using a probabilistic model of a dynamical system observed through a point process. In this model, the observation equation summarizes the stochastic properties of the observed heart beat point process while the essential features of the parasympathetic and sympathetic activity are concisely summarized in a history-dependent, autoregressive (AR), timevarying structure [64,65], allowing for definition of the instantaneous $R R$ and HR mean and variance, the instantaneous very low frequencies (VLF: 0 to $0.04 \mathrm{~Hz}$ ), low frequencies (LF: 0.04 to $0.15 \mathrm{~Hz}$ ), high frequencies (HF: 0.15 to $0.4 \mathrm{~Hz}$ ) indices in absolute $(p p V L F a, p p L F a, p p H F a)$ and normalized ( $p p L F u, p p H F u)$ spectral power, and the ratio between LF and HF ( $p p L F / H F)$. Vagal activity is the major contributor to the HF 
component. Some studies suggest that LF, when expressed in normalized units, is a quantitative marker of sympathetic modulation $[77,78]$, while other studies have found that LF reflects both sympathetic and vagal activity $[79,80]$. The LF/HF ratio is considered to reflect the sympathovagal balance. A thorough review of standard measures is presented in [32] and [81].

There are several advantages of using a point process framework to assess HRV: (a) the model overcomes stationarity requirements for standard HRV analysis; (b) previous methods compute similar estimates either on a beat-to-beat basis or in continuous time by preprocessing and filtering of the original $R-R$ interval series not justified by a physiological model of heart beat generation; (c) point process models include goodness-of-fit analyses supporting accurate description of the heartbeat stochastic structure; (d) point process instantaneous HRV indices can be computed simultaneously from a single statistical framework, they are computed in continuous time, and they can be extracted at any time resolution to track very fast dynamics; (e) our previous studies have shown that summaries comparable to standard HRV analysis (e.g., SDNN and LF/HF) can be obtained by averaging the instantaneous point process indices (static measures), which therefore encompass information from standard analysis; and (f) only instantaneous dynamics have been proved effective in characterizing central autonomic activity [82]. These results suggest that static and dynamic measures derived from point process models are good candidates for a more accurate, comprehensive description of ANS outflow dynamics $[64,65]$.

\section{Nonlinear assessment}

The point process framework also allows for inclusion of nonlinear structures and for definition of novel indices of nonlinear HRV dynamics, such as the bispectrum [67] and the instantaneous dominant Lyapunov exponent (IDLE) [75]. Results suggest that such quantification provides important information, which is independent from the standard autonomic assessment and is significantly correlated with loss of consciousness. In addition, we have demonstrated [67] that nonlinear indices such as detrended fluctuation analysis (DFA) and approximate entropy (ApEn) can be more accurately estimated by applying those methods to the instantaneous point process series, thus avoiding the need for long (e.g., $24 \mathrm{~h}$ ) recordings.

\section{RSA assessment}

We use an extension of the statistical point process model that we recently developed [68] to assess instantaneous estimates of respiratory sinus arrhythmia (RSA) from both ECG and respiration. Where respiratory information is available, we compute two RSA measures: the RSA gain at maximum coherence and RSA gain at maximum frequency. The instantaneous RSA assessments provide information that complements the standard HRV measures, particularly in the high frequency (HF) range, as well as indices such as the analgesia nociception index (ANI) and the surgical stress index (SSI) [53,56,57]. Importantly, only our new measures of RSA make it possible to reliably evaluate RSA in waning breathing conditions or whenever subjects show slow or unusual respiratory patterns [68]. 


\section{EEG assessment}

As described in detail in [15], we computed spectrograms using the multitaper method, implemented in the Chronux toolbox (http://chronux.org). The spectral analysis parameters were as follows: window length $T=4 \mathrm{~s}$ with 3-s overlap, time-bandwidth product $\mathrm{TW}=3$, number of tapers $K=5$. Although not shown, eigenvector decomposition analysis of the cross-spectral matrix was performed to identify the principal modes of oscillation. Further modal projection analysis characterized how power within these principal modes changed as a function of time. The relationship between lowfrequency phase $(0.1$ to $1 \mathrm{~Hz}$ ) and alpha/beta (8 to $14 \mathrm{~Hz}$ ) amplitude was extracted from the phase-amplitude histogram. These established EEG signatures help to characterize unconsciousness, track the transitions into and out of unconsciousness, and provide a means to monitor and predict the hypnotic state of patients under sedation and general anesthesia.

\section{Results}

\section{Tracking levels of consciousness}

The precise dynamics and relative timing of EEG changes during the transition between consciousness and unconsciousness have been recently described in previous works by Cimenser et al. and Purdon et al. [13-15]. These experiments found that responses to auditory stimuli show continuous changes in probability of response. Responses to salient stimuli (the sound of one's name) are lost later than responses to less salient stimuli (clicking noises). These features persist and strengthen with increasing concentrations of propofol and lead to burst suppression at sufficiently high doses. During emergence from anesthesia, this sequence reverses. Two patterns of phase-amplitude modulation were also discovered. The 'peak-max pattern' occurs in the unconscious state and is similar in character to slow oscillations observed during slow wave sleep. The 'troughmax pattern' is observed during the transitions to and from unconsciousness with propofol, whereas weaker and intermittent transitions into and out of unconsciousness are reported for DMED (see two exemplary spectrograms in Figure 1).

\section{Tracking ANS outflow dynamics}

Figure 2 shows tracking results from two recordings performed under the two considered studies, demonstrating how analysis of ANS outflow dynamics is able to provide critical information about autonomic effects by the two anesthetic agents. It is important to note that, as the two protocols have very different administration procedures, the tracking results are proposed together for methodological demonstration purposes and are not aimed at yielding specific comparative inferences between the two drugs.

In particular, PROP increases cardiovascular instability due to its hypotensive vasodilatatory effects, visible shortly after administration in mean RR, mean HR, ppHF, and ppLF. Hypotension usually requires further interventions such as, in the presented case, phenylephrine administration and assisted ventilation. These are mainly reflected in the respiratory coupling with heart rate (ppRSA). Excluding compensatory effects, a progressive long-term decrease along PROP administration in ppHF and ppLF suggests an increasingly poor autonomic tone, possibly associated with the significant baroreflex depression by PROP reported previously in Chen et al. [73]. The high hypnotic effect 


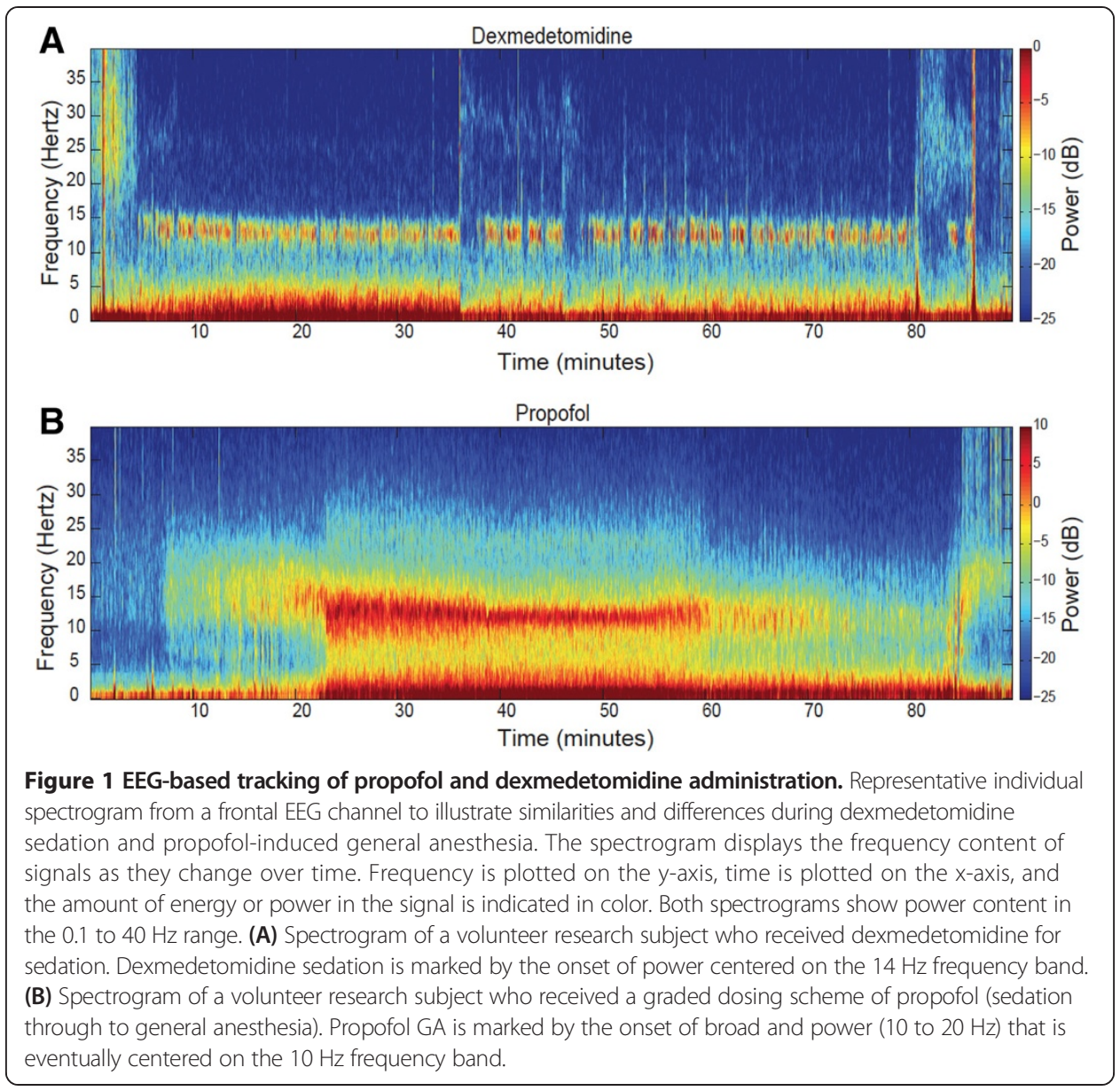

of PROP, leading to loss of consciousness as confirmed by EEG assessment, is reflected in the nonlinear IDLE index.

As a comparative example, the high bradycardic effect of DMED is clearly reflected in the RR and HR instantaneous assessment. ppHF increases as soon as DMED is administered and oscillates at significantly higher values, confirming the low sympathetic and predominant vagal activation effect. A sustained ppLF further confirms that baroreflex activation is maintained under administration [10]. On final note, respiratory frequency decreases and becomes more regular all along DMED administration. We further provide more details separately for each drug in the next paragraphs.

\section{The importance of using multimodal approaches, including respiration}

Figure 3 shows two examples focusing on the transition during DMED administration. These examples confirm that the mean RR and mean HR (Figure 3A,B) generally reflect a higher bradycardic effect during DMED administration, confirmed by the increase of the instantaneous vagally mediated HF HRV power (Figure 3C) and RSA gain (Figure 3F). Our results show that, generally, ppHF increases as soon as the drug is given (see example in Figure 3C), confirming the predominantly vagal activation effect. Such effect is not always observed, as shown in the second example (Figure 3G,H,I,J,K,L). In this case, the absence of the ppHF increase is clearly due to a progressive waning in respiratory variability (Figure 3L). 


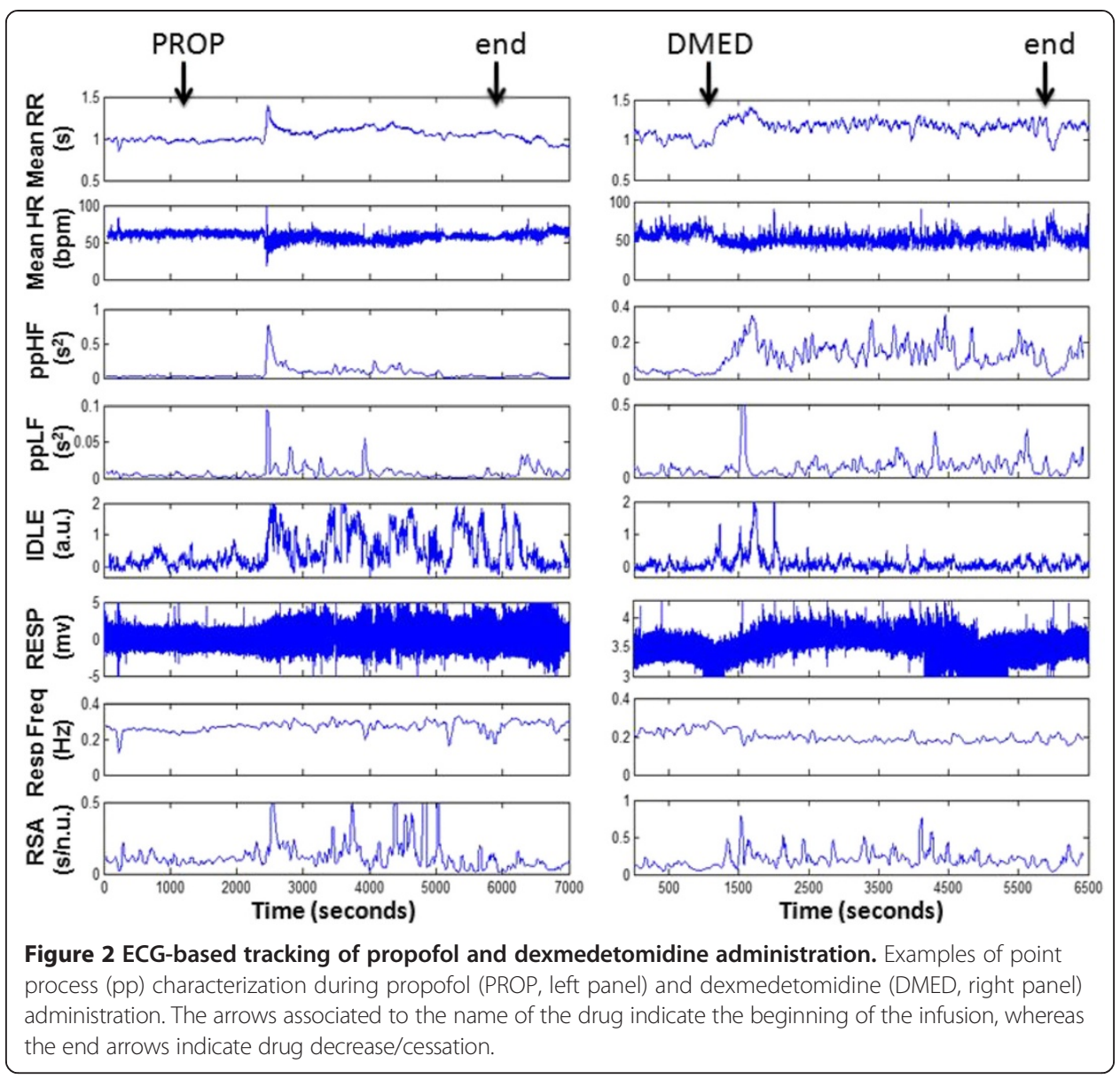

On the other hand, the RSA gain normalizes the RSA effect by respiratory power, as demonstrated by the increase in vagal activation in all subjects (including our examples in Figure 3F and 3L). Finally, it is important to note that respiration becomes more regular as drug administration progresses. This is validated by the lower variability of the respiratory rate around its mean value (see examples in Figure 3E,K).

\section{Towards instantaneous signatures of ANS dynamics during sedation}

In this section, we present a more detailed individual dynamics as function of the drug administration level, focusing on the linear and nonlinear HRV point process indices separately for each drug.

Although our aims are focused on the dynamics, we accompany the dynamical study with a brief statistical summary based on averaging our instantaneous indices. Tables 1 and 2 report median and median absolute deviation for the main instantaneous indices obtained from the ECG and averaged for each group and stages. The first segment is chosen within the baseline recording stage prior to the administration for both protocols (between 5 and $15 \mathrm{~min}$ ). Five levels are considered for PROP (15 min each). Two levels are considered for DMED: 8 min within the 10 min bolus administration (1 min after marker), and $18 \mathrm{~min}$ at low-level maintained administration (100 $\mathrm{s}$ after marker). To give a more multifaceted portrayal, in Figures 4 and 5, we are showing results from 


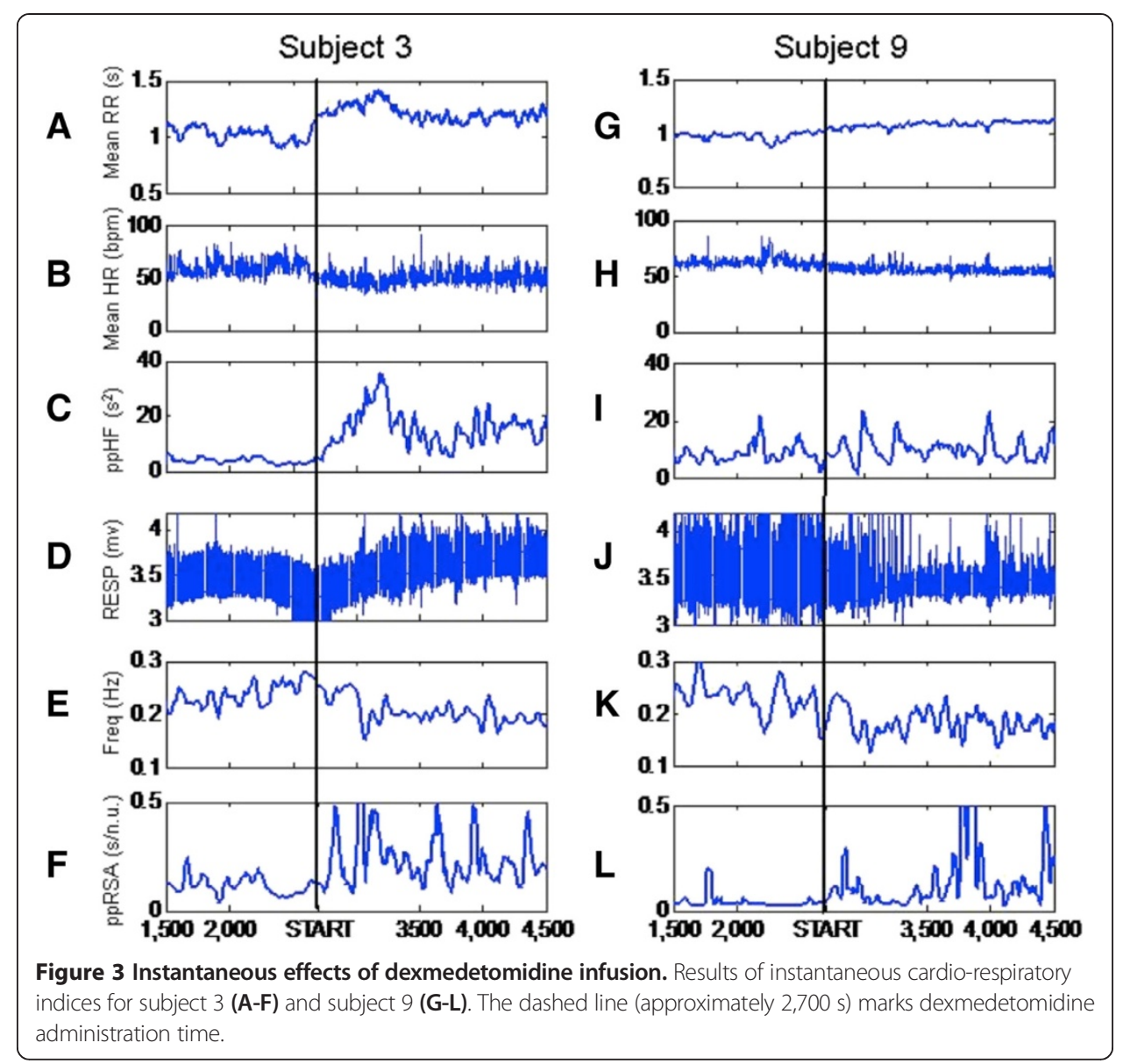

a single subject side to side with the dynamics averaged for all six subjects (appropriately aligned according to the experiment markers). The single subject indices give an idea on how single events other than drug administration changes affect the dynamics, whereas the averaged signatures, along with their confidence intervals (median absolute deviations), facilitate a clearer interpretation of the common changes due to change in drug levels as well as the degree of inter-subject variability for each index.

\section{Propofol signatures (Figure 4)}

These results pertain to the part of study 1 from baseline, to level 1 (L1), up to level 5 (L5), the highest level of PROP administration, and do not consider dynamics of emergence from anesthesia. In the subject portrayed on the left in Figure 4, the first three levels (baseline, L1, and L2) clearly confirm a sharp decrease in HRV, both in the LF and HF range, confirming the high decrease in autonomic tone, both vagal and sympathetic, with PROP. The relevant increase in variability at the beginning of level 3 is concomitant to administration of phenylephrine (arrow in the individual mean RR plot). In particular, the resulting vasoconstriction drives the autonomic balance towards parasympathetic action along the rest of the administration levels (bradycardia accompanied by high levels of ppHF together with a relevant, slow decrease in sympathovagal balance). Note that drug-induced physiological instability is not sensed by the nonlinear index IDLE even at the individual level, which maintains sustained levels up to mid- 
Table 1 Statistical analysis with propofol administration

\begin{tabular}{|c|c|c|c|c|c|c|}
\hline PROP & Baseline & L1 & L2 & L3 & L4 & L5 \\
\hline Mean RR (ms) & $948.9 \pm 186.1$ & $894.9 \pm 197.5$ & $880.3 \pm 170.2$ & $941.2 \pm 164.8$ & $1,047.4 \pm 166.6$ & $1,067.5 \pm 203.7$ \\
\hline Mean HR (bpm) & $63.7 \pm 11.88$ & $67.49 \pm 12.82$ & $68.49 \pm 13.66$ & $64.10 \pm 10.33$ & $58.07 \pm 9.12$ & $56.85 \pm 11.02$ \\
\hline $\operatorname{Var} \mathrm{RR}\left(\mathrm{ms}^{2}\right)$ & $1194.5 \pm$ & $486.44 \pm 366.47$ & $292.98 \pm 195.91$ & $1,100.2 \pm 851.37$ & $492.06 \pm 316.92$ & $1,397.6 \pm 1182.6$ \\
\hline Var HR (bpm²) & $2.50 \pm 1.22$ & $2.49 \pm 0.63$ & $1.46 \pm 0.97$ & $4.67 \pm 4.07$ & $2.57 \pm 1.87$ & $3.02 \pm 1.58$ \\
\hline $\mathrm{ppLF}\left(\mathrm{ms}^{2}\right)$ & $1,080.7 \pm 900.81$ & $499.31 \pm 309.57$ & $262.70 \pm 119.02$ & $1,202.9 \pm 1065.8$ & $934.04 \pm 478.23$ & $1,170.4 \pm 978.74$ \\
\hline ppLFn & $0.5834 \pm 0.0492$ & $0.5338 \pm 0.1334$ & $0.6508 \pm 0.1663$ & $0.7301 \pm 0.0510$ & $0.5635 \pm 0.2059$ & $0.4225 \pm 0.1452$ \\
\hline $\mathrm{ppHF}\left(\mathrm{ms}^{2}\right)$ & $941.83 \pm 273.91$ & $533.83 \pm 445.82$ & $237.49 \pm 163.70$ & $642.57 \pm 500.73$ & $299.24 \pm 97.62$ & $1,204.4 \pm 1056.7$ \\
\hline ppHFn & $0.4166 \pm 0.0492$ & $0.4662 \pm 0.1334$ & $0.3492 \pm 0.1663$ & $0.2699 \pm 0.0510$ & $0.4365 \pm 0.2059$ & $0.5775 \pm 0.1452$ \\
\hline LF/HF & $1.9064 \pm 0.6756$ & $1.0035 \pm 0.4972$ & $2.1198 \pm 1.2618$ & $2.8623 \pm 1.6639$ & $2.0781 \pm 1.5195$ & $0.9315 \pm 0.4612$ \\
\hline IDLE & $0.0085 \pm 0.0469$ & $0.0058 \pm 0.0607$ & $0.0391 \pm 0.0761$ & $0.1033 \pm 0.0342$ & $0.1262 \pm 0.0300$ & $0.1002 \pm 0.0801$ \\
\hline
\end{tabular}


Table 2 Statistical analysis with dexmedetomidine administration

\begin{tabular}{lccc}
\hline DMED & Baseline & Bolus & Maintenance \\
\hline Mean RR (ms) & $985.8 \pm 66.7$ & $1,067.3 \pm 101.4$ & $1,049.4 \pm 66.0$ \\
Mean HR $(\mathrm{bpm})$ & $61.48 \pm 4.09$ & $56.49 \pm 4.96$ & $57.51 \pm 3.55$ \\
Var RR $\left(\mathrm{ms}^{2}\right)$ & $899.78 \pm 284.96$ & $1,035.7 \pm 445.75$ & $467.09 \pm 193.95$ \\
Var HR $\left(\mathrm{bpm}^{2}\right)$ & $4.4436 \pm 0.6607$ & $3.3026 \pm 0.5365$ & $1.27 \pm 0.029$ \\
ppLF $\left(\mathrm{ms}^{2}\right)$ & $1,480.9 \pm 517.31$ & $1,573.2 \pm 465.20$ & $638.66 \pm 313.80$ \\
ppLFn & $0.5972 \pm 0.0918$ & $0.4340 \pm 0.2498$ & $0.5328 \pm 0.10381$ \\
ppHF $\left(\mathrm{ms}^{2}\right)$ & $849.45 \pm 223.67$ & $10.30 .1 \pm 409.63$ & $503.11 \pm 273.61$ \\
ppHFn & $0.4028 \pm 0.0918$ & $0.5660 \pm 0.2498$ & $0.4672 \pm 0.1038$ \\
LF/HF & $1.7550 \pm 0.8124$ & $0.9188 \pm 0.5079$ & $1.7286 \pm 0.5964$ \\
IDLE & $0.0409 \pm 0.0214$ & $0.1064 \pm 0.0825$ & $0.0242 \pm 0.0482$ \\
\hline
\end{tabular}

Median \pm median absolute deviation of the main instantaneous point process cardiovascular indices. Six subjects, DMED study 2.

level 4, and then it shows its most significant increase simultaneously with the reported loss of consciousness (arrow in the individual IDLE plot). The respective averaged signatures among all six subjects (Figure 4, right column) evidence the overall trends associated with the drug levels (appropriately aligned for each subject), thus 'blurring' the instantaneous effects of phenylephrine, which happens intermittently and at different times for each subjects. Of note, the measures with highest inter-individual variability, mirrored by a wider median absolute deviation region (in gray) are the mean RR and HR, followed by their respective variances. Conversely, the measures with least intersubject variability are the two normalized LF and HF powers and, most of all, the IDLE index of complexity. The IDLE is also showing the most coherent (increasing) trends associated with the level of drug when looking at the average and, at the same time, significant increases at the moment of loss of consciousness when looking at each subject individually.

\section{Dexmedetomidine signatures (Figure 5)}

These results pertain to the part of study 2 including baseline, 10 min of loading dose and $18 \mathrm{~min}$ of the following maintenance epoch. The marked bradycardic effect of the bolus dose is clear both at the individual and group level (increasing mean RR, decreasing mean HR). Bradycardia levels stabilize during maintenance. As the system is clearly migrating to a different state during loading, HRV (Var RR, Var HR, and ppLF) increases relevantly in the initial minutes and then tends to reach minimum levels by the end of the loading dose. On the other hand, ppHF increases at the start of administration and stays elevated for the entire $10 \mathrm{~min}$ of loading. As a consequence, the sympathovagal balance steadily decreases to minimum levels up to the end of the loading phase. The switch in balance during the maintenance period, along with HRV remaining at low levels, is quite probably due to the respiratory waning effect (not reportable for all subjects, see previous section for exemplary cases). Of note, the individual is losing consciousness at minute 8 of the loading epoch, regaining intermittent responsiveness from minute 11 after loading starts, whereas the average range of loss of consciousness for the group goes between 7 to 12 min after loading starts. Importantly, sharp increases in IDLE values can be observed around the range of loss of consciousness both for the individual and the group dynamics. 


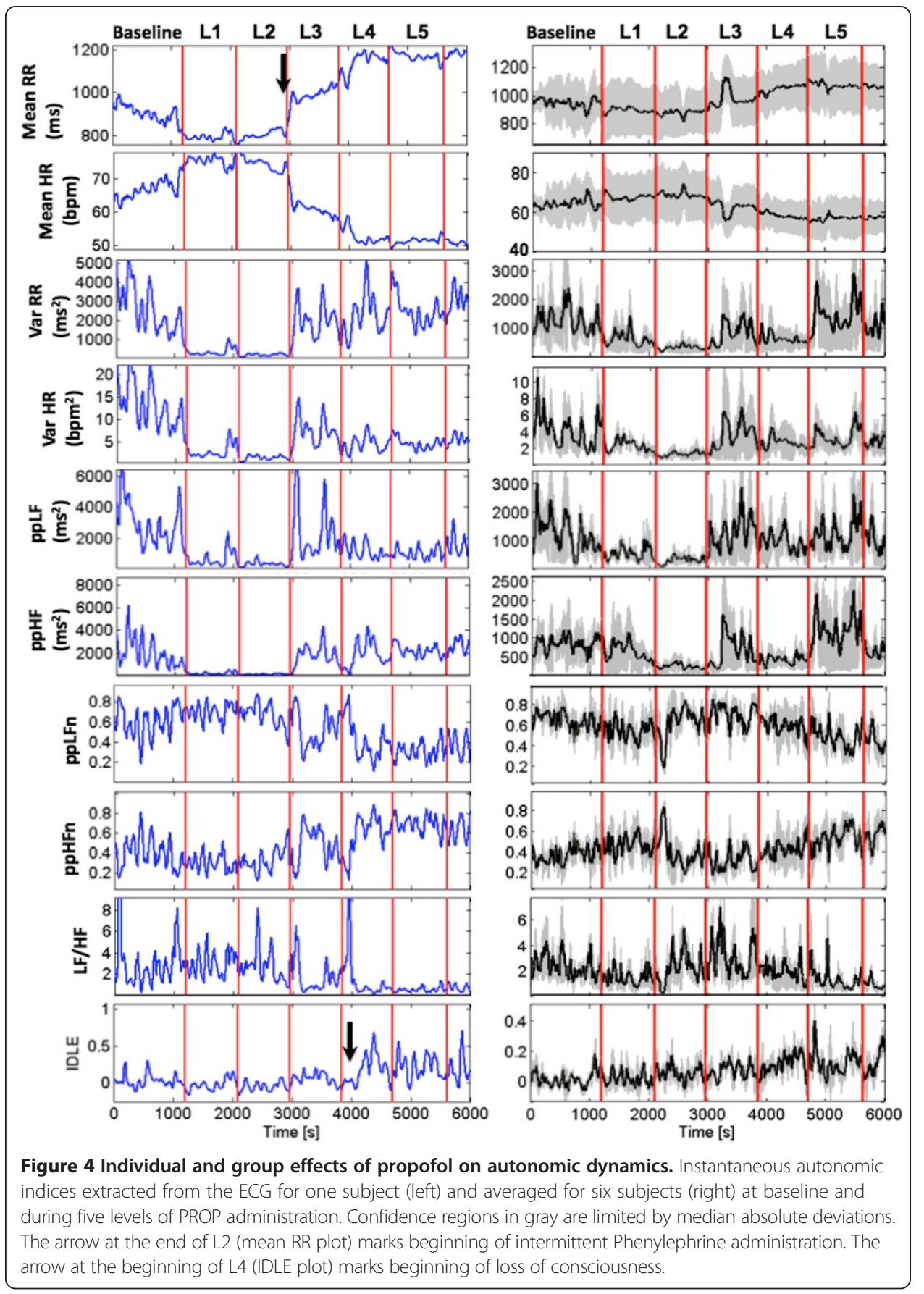

\section{Discussions and conclusions}

Our ongoing efforts are aimed at paving the way for a novel moment-to-moment ANS assessment in different states of sedation. Our main hypothesis is that different levels of sedation and analgesia affect the underlying neural processes and are reflected in different objective physiological signatures. As such, neural pathways are also differently affected, and specific physiological signatures of sedation could potentially be disentangled through appropriate experimental protocols and accurate noninvasive physiological assessments. 


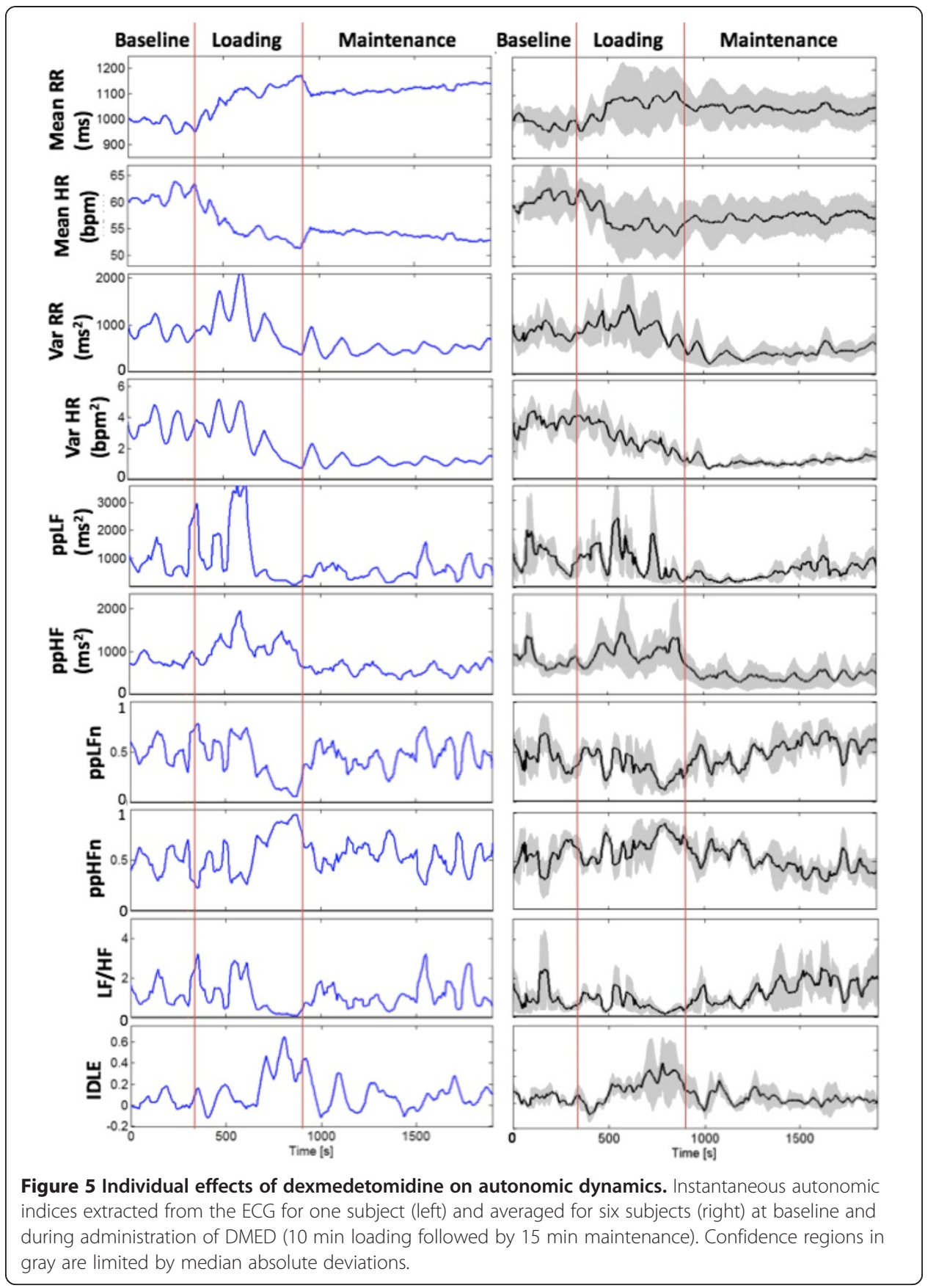

Within a multimodal framework including EEG, cardiovascular and respiratory assessment, we have devised a point process framework able to successfully characterize the variations in heartbeat dynamics when applied to PROP and DMED administration protocols. Previous results from our groups and other authors have stressed the importance of dynamic autonomic monitoring during anesthesia, and particularly during PROP and DMED $[13,15,19,20,34,44,46-48,51,53,54,72,73,75]$. In this presentation, we provide further evidence that our refined methods for analyzing the heartbeat dynamics during administration of specific anesthetic drugs are able to overcome nonstationary limitations, thus providing new real-time monitoring approaches to patients receiving anesthesia. 
In particular, we show the application of instantaneous linear and nonlinear estimates of heartbeat dynamics as measures defined in the time and frequency domain as well as the instantaneous dominant Lyapunov exponent [75,83-85]. As a result, our framework is able to examine the complex evolution of the unevenly sampled heartbeat dynamics series during anesthesia and sedation, in continuous time without the use of interpolation filters. Remarkably, most other nonlinearity and complexity indices are derived from non-parametric models, whereas our model is purely parametric and the analytically derived indices can be evaluated in a dynamic and instantaneous fashion. The proposed framework also allows for the inclusion of covariates as the respiration activity, thus being able to estimate other meaningful measures as the instantaneous RSA. We believe these strengths enable our method as a useful anesthesia and sedation assessment tool taking into account also the nonlinear dynamics of heartbeat intervals in a highly non-stationary environment. Moreover, goodness of fit measures such as Kolmogorov-Smirnov (KS) distance and autocorrelation plots quantitatively allow to verify the model fit as well as to choose the proper model order, which represents another open issue of current parametric approaches.

In the results from the first experimental study, we show that PROP signatures are initially (first two administration levels) characterized by a marked decrease in HRV, both in LF and HF, confirming previous findings, and pointing at a general loss of autonomic tone (both sympathetic and vagal) possibly connected with simultaneous baroreflex deactivation/resetting. The observed delayed compensatory variations of HRV can be attributed to sympathetic activation due to vasodilatory effect, as well as vagal activation due to intermittent phenylephrine administration. Vagal predominance is observed during loss of consciousness. Our comparison between individual signatures and signatures obtained from all subjects demonstrates that overall trends, devoid of fast transient effect present on individual dynamics, can be observed particularly in normalized measures. Most importantly, increased IDLE complex dynamics elicited by increasing drug administration levels are highly correlated with the hypnotic effect (as measured by auditory test and confirmed by EEG metrics) for the individual and with the level of administration for the group signature. In the results from the second experimental study, we show that DMED signatures are characterized by a marked sympathetic deactivation and by sustained vagal activation, clearly visible in HRV dynamics during the first loading stage, so less during maintenance levels, where respiration waning effects are more predominant. Importantly, the IDLE complex dynamic increases in accordance with the incidence of loss of consciousness (predominantly during the initial bolus administration). The reduced hypnotic effect compared with PROP (also demonstrated by EEG signatures) is also confirmed by relatively low IDLE levels during maintenance. Although we do not provide $p$ values from statistical inference tests (because of the reduced number of subjects involved in the study), we show consistent trends in all the linear and nonlinear heartbeat features (see Figures 4 and 5) and, thus, provide important insights to the different cardiovascular dynamics during anesthesia and sedation as shown in an instantaneous fashion.

The main challenge of the proposed study, and all HRV studies in general, is the high inter-individual variability, mainly due to the complexity of the cardiovascular control responses to intrinsic or induced perturbations of the system, particularly with drug administration. Such variability has been the prominent limitation preventing previous 
studies to go beyond statistical summaries indicating only general trends within a limited population, and to provide a personalized tracking of sedation. Moreover, another limitation of our methodology can be related to the need of a preliminary calibration phase before it can be effectively used to estimate the instantaneous measures. Like other parametric methods, in fact, a tuning of model parameters such as model order and time-window $W$ size for the local-likelihood parameter estimation. To this extent, in the presented application, we were able to obtain reproducible and reliable results by using standard values such a $W=90 \mathrm{~s}$, as well as optimal model orders by minimizing the KS statistics.

Given these limitations, we will center our future studies on two principles: (a) the high variability reflected in the resulting statistical predictions, and physiological interpretations must be accompanied by a multiorgan approach and a careful choice of complementary information; and (b) a powerful and reliable classification algorithm is required to use dimensionality towards optimal discrimination. We believe that validation of accurate and reliable scales based on the instantaneous identification, together with the careful choices in sedation levels (which are the results of our extensive preliminary investigations) will provide a more sensitive assessment and interpretation of the results. In particular, differently than previous investigations, we will consider EEG and HRV measures together and feed them to a classifier to find the most efficient combination signature. Most importantly, we will be able to consider a novel measure of RSA, which accounts for respiratory pattern variations in assessing sympathovagal dynamics. Our future studies will determine by which degree a combined index of the ANS measures is able to accurately quantify sedation in controlled scenarios, also estimating recently proposed instantaneous nonlinear measures based on high-order spectral analysis and entropy [70,83-90]. We will devise classifiers which might provide enough power to produce a combination of measures of autonomic outflow validating the assessment for each single subject, thus paving the way for the feasibility for a realtime monitoring tool able to track sedation in uncontrolled scenarios.

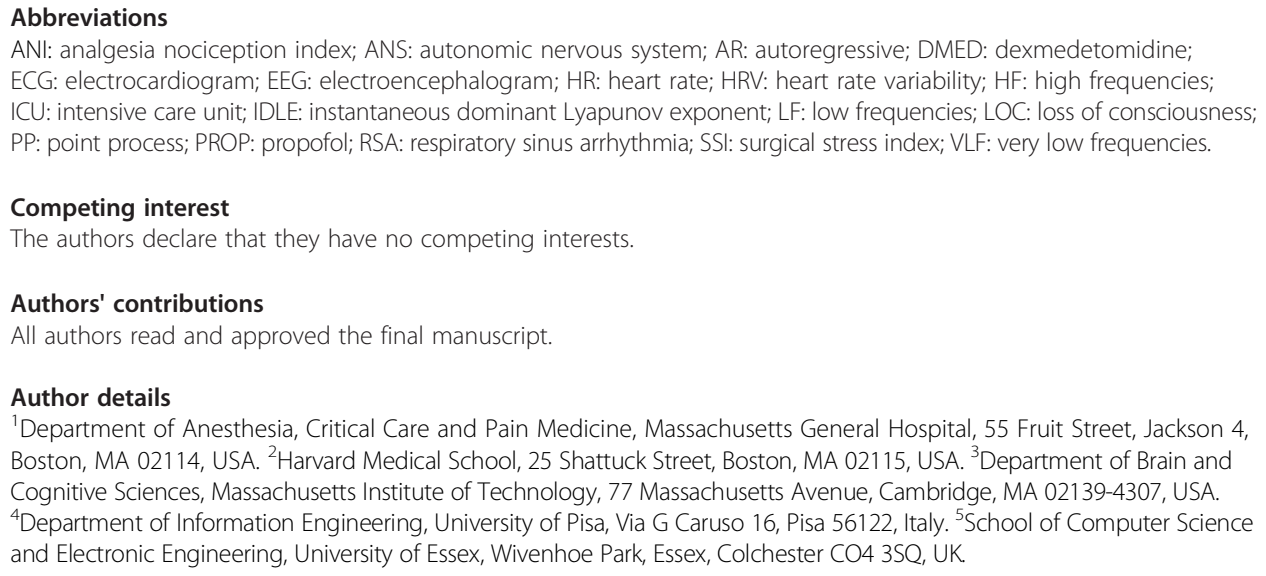

1. Ghoneim MM, Block Rl: Learning and memory during general anesthesia: an update. Anesthesiology 1997 87:387-410

2. Sackel DJ: Anesthesia awareness: an analysis of its incidence, the risk factors involved, and prevention. $J$ Clin Anesth 2006, 18:483-485. 
3. Mhuircheartaigh RN, Rosenorn-Lanng D, Wise R, Jbabdi S, Rogers R, Tracey I: Cortical and subcortical connectivity changes during decreasing levels of consciousness in humans: a functional magnetic resonance imaging study using propofol. J Neurosci 2010, 30:9095-9102.

4. Lin S, Quan X, Zou L, Ye T: Effects of propofol on brain activation in respond to mechanical stimuli. Zhongguo Yi Xue Ke Xue Yuan Xue Bao 2012, 34:222-227.

5. Hofbauer RK, Fiset P, Plourde G, Backman SB, Bushnell MC: Dose-dependent effects of propofol on the central processing of thermal pain. Anesthesiology 2004, 100:386-394.

6. Brown EN, Lydic R, Schiff ND: General anesthesia, sleep, and coma. N Engl J Med 2010, 363:2638-2650.

7. Kearse LA Jr, Brown EN, McPeck K: Somatosensory evoked potentials sensitivity relative to electroencephalography for cerebral ischemia during carotid endarterectomy. Stroke 1992, 23:498-505.

8. Palanca BJA, Mashour GA, Avidan MS: Processed electroencephalogram in depth of anesthesia monitoring. Curr Opin Anaesthesiol 2009, 22:553-559.

9. Tinker JH, Sharbrough FW, Michenfelder JD: Anterior shift of the dominant EEG rhythm during anesthesia in the Java monkey: correlation with anesthetic potency. Anesthesiology 1977, 46:252-259.

10. Gugino LD, Chabot RJ, Prichep LS, John ER, Formanek V, Aglio LS: Quantitative EEG changes associated with loss and return of consciousness in healthy adult volunteers anaesthetized with propofol or sevoflurane. Br J Anaesth 2001, 87:421-428.

11. John ER, Prichep LS, Kox W, Valdés-Sosa P, Bosch-Bayard J, Aubert E, Tom M, di Michele F, Gugino LD, diMichele F: Invariant reversible QEEG effects of anesthetics. Conscious Cogn 2001, 10:165-183.

12. Feshchenko VA, Veselis RA, Reinsel RA: Propofol-induced alpha rhythm. Neuropsychobiology 2004, 50:257-266.

13. Cimenser A, Purdon PL, Pierce ET, Walsh JL, Salazar-Gomez AF, Harrell PG, Tavares-Stoeckel C, Habeeb K, Brown EN: Tracking brain states under general anesthesia by using global coherence analysis. Proc Natl Acad Sci U S A 2011, 108:8832-8837.

14. Purdon PL, Pierce ET, Bonmassar G, Walsh J, Harrell PG, Kwo J, Deschler D, Barlow M, Merhar RC, Lamus C, Mullaly CM, Sullivan M, Maginnis S, Skoniecki D, Higgins H-A, Brown EN: Simultaneous electroencephalography and functional magnetic resonance imaging of general anesthesia. Ann N Y Acad Sci 2009, 1157:61-70.

15. Purdon PL, Pierce ET, Mukamel EA, Prerau MJ, Walsh JL, Wong KFK, Salazar-Gomez AF, Harrell PG, Sampson AL, Cimenser A, Ching S, Kopell NJ, Tavares-Stoeckel C, Habeeb K, Merhar R, Brown EN: Electroencephalogram signatures of loss and recovery of consciousness from propofol. Proc Natl Acad Sci U S A 2013, 110:E1142-E1151.

16. Rampil IJ, Matteo RS: Changes in EEG spectral edge frequency correlate with the hemodynamic response to laryngoscopy and intubation. Anesthesiology 1987, 67:139-142

17. Supp GG, Siegel M, Hipp JF, Engel AK: Cortical hypersynchrony predicts breakdown of sensory processing during loss of consciousness. Curr Biol 2011, 21:1988-1993.

18. Jäntti V, Yli-Hankala A, Baer GA, Porkkala T: Slow potentials of EEG burst suppression pattern during anaesthesia. Acta Anaesthesiol Scand 1993, 37:121-123.

19. Sato M, Tanaka M, Umehara S, Nishikawa T: Baroreflex control of heart rate during and after propofol infusion in humans. Br J Anaesth 2005, 94:577-581.

20. Dorantes Mendez G, Aletti F, Toschi N, Canichella A, Dauri M, Coniglione F, Guerrisi M, Signorini MG, Cerutti S, Ferrario M: Baroreflex sensitivity variations in response to propofol anesthesia: comparison between normotensive and hypertensive patients. J Clin Monit Comput 2013, 27:417-426.

21. Ebert TJ, Muzi M: Propofol and autonomic reflex function in humans. Anesth Analg 1994, 78:369-375.

22. Arain SR, Ebert TJ: The efficacy, side effects, and recovery characteristics of dexmedetomidine versus propofol when used for intraoperative sedation. Anesth Analg 2002, 95:461-466. table of contents.

23. Penttilä J, Helminen A, Anttila M, Hinkka S, Scheinin H: Cardiovascular and parasympathetic effects of dexmedetomidine in healthy subjects. Can J Physiol Pharmacol 2004, 82:359-362.

24. Hogue CW Jr, Talke P, Stein PK, Richardson C, Domitrovich PP, Sessler DI: Autonomic nervous system responses during sedative infusions of dexmedetomidine. Anesthesiology 2002, 97:592-598.

25. Belleville JP, Ward DS, Bloor BC, Maze M: Effects of intravenous dexmedetomidine in humans. I. Sedation, ventilation, and metabolic rate. Anesthesiology 1992, 77:1125-1133.

26. Bloor BC, Ward DS, Belleville JP, Maze M: Effects of intravenous dexmedetomidine in humans. II. Hemodynamic changes. Anesthesiology 1992, 77:1134-1142.

27. Cortinez LI, Hsu Y-W, Sum-Ping ST, Young C, Keifer JC, Macleod D, Robertson KM, Wright DR, Moretti EW, Somma J: Dexmedetomidine pharmacodynamics: part II: crossover comparison of the analgesic effect of dexmedetomidine and remifentanil in healthy volunteers. Anesthesiology 2004, 101:1077-1083.

28. Ehara T, Ogawa Y, Kato J, Aoki K, Ogawa S, Iwasaki K: The effect of dexmedetomidine on arterial-cardiac baroreflex function assessed by spectral and transfer function analysis. J Anesth 2012, 26:483-489.

29. Feld J, Hoffman WE, Paisansathan C, Park H, Ananda RC: Autonomic activity during dexmedetomidine or fentanyl infusion with desflurane anesthesia. J Clin Anesth 2007, 19:30-36.

30. Hsu Y-W, Cortinez LI, Robertson KM, Keifer JC, Sum-Ping ST, Moretti EW, Young CC, Wright DR, Macleod DB, Somma J: Dexmedetomidine pharmacodynamics: part l: crossover comparison of the respiratory effects of dexmedetomidine and remifentanil in healthy volunteers. Anesthesiology 2004, 101:1066-1076.

31. Hu R, Liu JX, Jiang H: Dexmedetomidine versus remifentanil sedation during awake fiberoptic nasotracheal intubation: a double-blinded randomized controlled trial. J Anesth 2012, 27:211-217.

32. Task Force of the European Society of Cardiology and the North American Society of Pacing and Electrophysiology: Heart rate variability: standards of measurement, physiological interpretation and clinical use. Circulation 1996, 93:1043-1065.

33. Riznyk L, Fijałkowska M, Przesmycki K: Effects of thiopental and propofol on heart rate variability during fentanyl-based induction of general anesthesia. Pharmacol Rep 2005, 57:128-134.

34. Mazzeo AT, La Monaca E, Di Leo R, Vita G, Santamaria LB: Heart rate variability: a diagnostic and prognostic tool in anesthesia and intensive care. Acta Anaesthesiol Scand 2011, 55:797-811. 
35. Scheffer GJ, Ten Voorde BJ, Karemaker JM, Ros HH, de Lange JJ: Effects of thiopentone, etomidate and propofol on beat-to-beat cardiovascular signals in man. Anaesthesia 1993, 48:849-855.

36. Galletly DC, Westenberg AM, Robinson BJ, Corfiatis T: Effect of halothane, isoflurane and fentanyl on spectral components of heart rate variability. Br J Anaesth 1994, 72:177-180.

37. Marsch SC, Skarvan K, Schaefer HG, Naegeli B, Paganoni R, Castelli I, Scheidegger D: Prolonged decrease in heart rate variability after elective hip arthroplasty. Br J Anaesth 1994, 72:643-649.

38. Ireland N, Meagher J, Sleigh JW, Henderson JD: Heart rate variability in patients recovering from general anaesthesia. Br J Anaesth 1996, 76:657-662.

39. Huang HH, Chan HL, Lin PL, Wu CP, Huang CH: Time-frequency spectral analysis of heart rate variability during induction of general anaesthesia. Br J Anaesth 1997, 79:754-758.

40. Schubert A, Palazzolo JA, Brum JM, Ribeiro MP, Tan M: Heart rate, heart rate variability, and blood pressure during perioperative stressor events in abdominal surgery. J Clin Anesth 1997, 9:52-60.

41. Blues $C M$, Pomfrett $\mathrm{CJ}$ : Respiratory sinus arrhythmia and clinical signs of anaesthesia in children. $\mathrm{Br} J$ Anaesth 1998, 81:333-337.

42. Paris A, Tonner PH, Bein B, von Knobelsdorff G, Scholz J: [Heart rate variability in anesthesia]. Anaesthesiol Reanim 2001, 26:60-69.

43. Larsen PD, Galletly DC: Cardioventilatory coupling in heart rate variability: the value of standard analytical techniques. Br J Anaesth 2001, 87:819-826.

44. Pichot V, Buffière S, Gaspoz JM, Costes F, Molliex S, Duverney D, Roche F, Barthélémy JC: Wavelet transform of heart rate variability to assess autonomic nervous system activity does not predict arousal from general anesthesia. Can J Anaesth 2001, 48:859-863.

45. Kanaya N, Hirata N, Kurosawa S, Nakayama M, Namiki A: Differential effects of propofol and sevoflurane on heart rate variability. Anesthesiology 2003, 98:34-40.

46. Ogawa Y, Iwasaki K, Shibata S, Kato J, Ogawa S, Oi Y: Different effects on circulatory control during volatile induction and maintenance of anesthesia and total intravenous anesthesia: autonomic nervous activity and arterial cardiac baroreflex function evaluated by blood pressure and heart rate variability analysis. J Clin Anesth 2006, 18:87-95.

47. Ledowski T, Bein B, Hanss R, Paris A, Fudickar W, Scholz J, Tonner PH: Neuroendocrine stress response and heart rate variability: a comparison of total intravenous versus balanced anesthesia. Anesth Analg 2005, 101:1700-1705.

48. Win NN, Fukayama $\mathrm{H}$, Kohase $\mathrm{H}$, Umino M: The different effects of intravenous propofol and midazolam sedation on hemodynamic and heart rate variability. Anesth Analg 2005, 101:97-102. table of contents.

49. Luginbühl M, Yppärilä-Wolters H, Rüfenacht M, Petersen-Felix S, Korhonen I: Heart rate variability does not discriminate between different levels of haemodynamic responsiveness during surgical anaesthesia. Br J Anaesth 2007, 98:728-736

50. Tarvainen MP, Georgiadis S, Lipponen JA, Laitio T, Karjalainen PA, Scheinin H, Kaskinoro K: Analysis of heart rate variability dynamics during propofol and dexmedetomidine anesthesia. Conf Proc IEEE Eng Med Biol Soc 2010, 2010:1634-1637

51. Tarvainen MP, Georgiadis S, Laitio T, Lipponen JA, Karjalainen PA, Kaskinoro K, Scheinin H: Heart rate variability dynamics during low-dose propofol and dexmedetomidine anesthesia. Ann Biomed Eng 2012, 40:1802-1813.

52. Balocchi R, Varanini M, Menicucci D, Santarcangelo EL, Migliorini S, Fontani G, Carli G: Heart rate variability in subjects with different hypnotic susceptibility receiving nociceptive stimulation and suggestions of analgesia. Conf Proc IEEE Eng Med Biol Soc 2005, 7:6996-6999.

53. Huiku M, Uutela K, van Gils M, Korhonen I, Kymäläinen M, Meriläinen P, Paloheimo M, Rantanen M, Takala P, Viertiö-Oja H, Yli-Hankala A: Assessment of surgical stress during general anaesthesia. Br J Anaesth 2007, 98:447-455.

54. Jeanne M, Logier R, De Jonckheere J, Tavernier B: Validation of a graphic measurement of heart rate variability to assess analgesia/nociception balance during general anesthesia. Conf Proc IEEE Eng Med Biol Soc 2009, 2009:1840-1843

55. Huhle R, Burghardt M, Zaunseder S, Wessel N, Koch T, Malberg H, Heller AR: Effects of awareness and nociception on heart rate variability during general anaesthesia. Physiol Meas 2012, 33:207-217.

56. Jeanne M, Clément C, De Jonckheere J, Logier R, Tavernier B: Variations of the analgesia nociception index during general anaesthesia for laparoscopic abdominal surgery. J Clin Monit Comput 2012, 26:289-294.

57. Gruenewald M, llies C, Herz J, Schoenherr T, Fudickar A, Höcker J, Bein B, Gruenewald M, llies C, Herz J, Schoenher T, Fudickar A, Höcker J, Bein B: Influence of nociceptive stimulation on analgesia nociception index (ANI) during propofol-remifentanil anaesthesia. Br J Anaesth 2013, 110:1024-1030.

58. Latson TW, O'Flaherty D: Effects of surgical stimulation on autonomic reflex function: assessment by changes in heart rate variability. Br J Anaesth 1993, 70:301-305.

59. Ledowski T, Tiong WS, Lee C, Wong B, Fiori T, Parker N: Analgesia nociception index: evaluation as a new parameter for acute postoperative pain. Br J Anaesth 2013, 111:627-629.

60. Wallin BG, König U: Changes of skin nerve sympathetic activity during induction of general anaesthesia with thiopentone in man. Brain Res 1976, 103:157-160.

61. Bergmann I, Göhner A, Crozier TA, Hesjedal B, Wiese CH, Popov AF, Bauer M, Hinz JM: Surgical pleth indexguided remifentanil administration reduces remifentanil and propofol consumption and shortens recovery times in outpatient anaesthesia. Br J Anaesth 2013, 110:622-628.

62. Bonhomme V, Uutela K, Hans G, Maquoi I, Born JD, Brichant JF, Lamy M, Hans P: Comparison of the surgical Pleth Index ${ }^{\mathrm{TM}}$ with haemodynamic variables to assess nociception-anti-nociception balance during general anaesthesia. Br J Anaesth 2011, 106:101-111.

63. Ilies C, Gruenewald M, Ludwigs J, Thee C, Höcker J, Hanss R, Steinfath M, Bein B: Evaluation of the surgical stress index during spinal and general anaesthesia. Br J Anaesth 2010, 105:533-537.

64. Barbieri R, Matten EC, Alabi AA, Brown EN: A point-process model of human heartbeat intervals: new definitions of heart rate and heart rate variability. Am J Physiol Heart Circ Physiol 2005, 288:H424-H435. 
65. Barbieri R, Brown EN: Analysis of heartbeat dynamics by point process adaptive filtering. IEEE Trans Biomed Eng 2006, 53:4-12.

66. Chen Z, Brown EN, Barbieri R: Assessment of autonomic control and respiratory sinus arrhythmia using point process models of human heart beat dynamics. IEEE Trans Biomed Eng 2009, 56:1791-1802.

67. Chen Z, Brown E, Barbieri R: Characterizing nonlinear heartbeat dynamics within a point process framework. IEEE Trans Biomed Eng 2010, 2008:2781-2784

68. Kodituwakku S, Lazar SW, Indic P, Chen Z, Brown EN, Barbieri R: Point process time-frequency analysis of dynamic respiratory patterns during meditation practice. Med Biol Eng Comput 2012, 50:261-275.

69. Chen Z, Brown EN, Barbieri R: A unified point process probabilistic framework to assess heartbeat dynamics and autonomic cardiovascular control. Front Physio 2012, 3:4.

70. Valenza G, Citi L, Scilingo EP, Barbieri R: Point-process nonlinear models with Laguerre and Volterra expansions: instantaneous assessment of heartbeat dynamics. IEEE Trans Signal Process 2013, 61:2914-2926.

71. Chen Z, Purdon P, Pierce E, Harrell G, Brown E, Barbieri R: Assessment of baroreflex control of heart rate during general anesthesia using a point process method. Proc IEEE Int Conf Acoust Speech Signal Process ICASSP 2009, 2009:333-336.

72. Chen Z, Purdon PL, Pierce ET, Harrell G, Walsh J, Salazar AF, Tavares CL, Brown EN, Barbieri R: Linear and nonlinear quantification of respiratory sinus arrhythmia during propofol general anesthesia. Conf Proc IEEE Eng Med Biol Soc 2009, 2009:5336-5339.

73. Chen Z, Purdon PL, Harrell G, Pierce ET, Walsh J, Brown EN, Barbieri R: Dynamic assessment of baroreflex control of heart rate during induction of propofol anesthesia using a point process method. Ann Biomed Eng 2011, 39:260-276

74. Chen Z, Citi L, Purdon PL, Brown EN, Barbieri R: Instantaneous assessment of autonomic cardiovascular control during general anesthesia. Conf Proc IEEE Eng Med Biol Soc 2011, 2011:8444-8447.

75. Citi L, Valenza G, Purdon PL, Brown EN, Barbieri R: Monitoring heartbeat nonlinear dynamics during general anesthesia by using the instantaneous dominant Lyapunov exponent. Conf Proc IEEE Eng Med Biol Soc 2012, 2012:3124-3127.

76. Citi L, Brown EN, Barbieri R: A real-time automated point-process method for the detection and correction of erroneous and ectopic heartbeats. leee Trans Biomed Eng 2012, 59:2828-2837.

77. Hirsch JA, Bishop B: Respiratory sinus arrhythmia in humans: how breathing pattern modulates heart rate. Am J Physiol 1981, 241:H620-H629.

78. Malliani A, Pagani M, Lombardi F, Cerutti S: Cardiovascular neural regulation explored in the frequency domain Circulation 1991, 84:482-492.

79. Di Rienzo M, Castiglioni P, Mancia G, Parati G, Pedotti A: 24 h sequential spectral analysis of arterial blood pressure and pulse interval in free-moving subjects. IEEE Trans Biomed Eng 1989, 36:1066-1075.

80. Pomeranz B, Macaulay RJ, Caudill MA, Kutz I, Adam D, Gordon D, Kilborn KM, Barger AC, Shannon DC, Cohen RJ Assessment of autonomic function in humans by heart rate spectral analysis. Am J Physiol 1985, 248(1 Pt 2):H151-H153.

81. Fallen EL, Kamath MV, Ghista DN, Fitchett D: Spectral analysis of heart rate variability following human heart transplantation: evidence for functional reinnervation. J Auton Nerv Syst 1988, 23:199-206.

82. Napadow V, Dhond R, Conti G, Makris N, Brown EN, Barbieri R: Brain correlates of autonomic modulation: combining heart rate variability with fMRI. Neuroimage 2008, 42:169-177.

83. Citi L, Valenza G, Barbieri R: Instantaneous estimation of high-order nonlinear heartbeat dynamics by Lyapunov exponents. Conf Proc IEEE Eng Med Biol Soc 2012, 2012:13-16.

84. Valenza G, Citi L, Lanata A, Scilingo EP, Barbieri R: A nonlinear heartbeat dynamics model approach for personalized emotion recognition. Conf Proc IEEE Eng Med Biol Soc 2013, 2013:2579-2582.

85. Valenza G, Citi L, Barbieri R: Instantaneous nonlinear assessment of complex cardiovascular dynamics by Laguerre-Volterra point process models. Conf Proc IEEE Eng Med Biol Soc 2013, 2013:6131-6134.

86. Valenza G, Citi L, Scilingo EP, Barbieri R: Using Laguerre expansion within point-process models of heartbeat dynamics: a comparative study. Conf Proc IEEE Eng Med Biol Soc 2012, 2012:29-32.

87. Valenza G, Citi L, Gentili C, Lanatá A, Scilingo EP, Barbieri R: Point-process nonlinear autonomic assessment of depressive states in bipolar patients. Methods Inf Med 2014, 53:296-302.

88. Valenza G, Citi L, Lanatá A, Scilingo EP, Barbieri R: Revealing real-time emotional responses: a personalized assessment based on heartbeat dynamics. Sci Rep 2014, 4:4998.

89. Valenza G, Citi L, Barbieri R: Estimation of instantaneous complex dynamics through Lyapunov exponents: a study on heartbeat dynamics. PLOS ONE 2014, 9(8):e105622.

90. Valenza G, Citi L, Scilingo EP, Barbieri R: Inhomogeneous point-process entropy: an instantaneous measure of complexity in discrete systems. Phys Rev E 2014, 89:052803.

doi:10.1186/s40244-014-0013-2

Cite this article as: Valenza et al:. Instantaneous monitoring of heart beat dynamics during anesthesia and sedation. Journal of Computational Surgery 2014 1:13. 